TPI-MINN-95-19/T

NUC-MINN-95-15/T

HEP-MINN-95-1344

26 July 1995

\title{
Magnetic Fields Produced by Phase Transition Bubbles in the Electroweak Phase Transition
}

\author{
Gordon Baym \\ Department of Physics, University of Illinois at Urbana-Champaign, Urbana, IL 61801 \\ Dietrich Bödeker and Larry McLerran \\ School of Physics and Astronomy, University of Minnesota, Minneapolis, MN 55455
}

(July 23, 2018)

\begin{abstract}
The electroweak phase transition, if proceeding through nucleation and growth of bubbles, should generate large scale turbulent flow, which in turn generates magnetic turbulence and hence magnetic fields on the scale of turbulent flow. We discuss the seeding of this turbulent field by the motion of the dipole charge layers in the phase transition bubble walls, and estimate the strength of the produced fields.
\end{abstract}

\section{INTRODUCTION}

The problem of the generation of magnetic fields is an old one in cosmology [1],2]. The general approach is to identify mechanisms for the generation of seed fields [3]- [7], which can become amplified into fields on galactic scales. Considerable attention has been focused 
on generation of seed fields during cosmic phase transitions, e.g., Ref. [幽, particularly at the electroweak transition [5], or at the QCD transition through formation of electromagnetic fields in the collision of shock fronts [6] or formation of dipole charge layers on the surfaces of phase transition bubble walls whose motion generates an electromagnetic current and hence a magnetic field [7]. Always present as a source of seed fields are random magnetic field fluctuations on a scale of order a thermal wavelength. Once one has identified the source of microscale seed fields, it is necessary to find how they are transformed into macroscopic scale magnetic fields, a process requiring production of current loops on large size scales, and thus the generation of a level of vorticity in the fluid.

In this paper we focus on the generation and amplification of fields at the electroweak phase transition, by means of the fluid, and hence magnetic, turbulence which should be formed there. During a first-order electroweak phase transition bubbles of the new phase form [8]- [12] [but see arguments in Ref. [13] why the transition, even if first order transition, need not necessarily proceed dynamically via domain wall formation]. Then, as we argue in Sec. II, bubble collisions generate a level of turbulence and hence vorticity in the fluid. As a bubble propagates through the electroweak plasma, it generates a precursor shock front which accelerates the fluid outside the burning front of the bubble. As the shock fronts from different bubbles collide, they generate turbulent jets on a scale of order the size of a typical bubble. The turbulence in the fluid amplifies whatever seed fields are present to finite-amplitude large-scale size magnetic fields [14]. These fields can be considerable, if there is time for equipartition between the kinetic energy associated with the turbulent flow and the magnetic field energy.

A possible mechanism of generation of magnetic seed fields is via the dipole electromagnetic charge layer on the surfaces of the bubbles that forms, as discussed in Sec. III, as a consequence of baryon asymmetry and the large mass of the top quark. The burning surfaces of bubbles propagating within a region of turbulent flow rotate in the moving fluid. The rotation of the dipole charge layer thus sets up a current in the fluid. We estimate the typical magnetic field generated by a single bubble, and then argue that these fields provide 
at least one source of seed magnetic fields in the turbulent fluid which are then amplified into full scale magnetic turbulence. The strength of the fields produced this way are considerably smaller in the absence of magnetic turbulence than the fields that would result from equipartition in the turbulent flow.

In order to estimate the generated magnetic field on scale sizes large compared to the electroweak scale through the cumulative effect of the many bubbles in the system, we follow the classic analysis of Hogan [4], and determine, in Sec. IV, the magnetic field correlation function,

$$
C(\mathbf{r})=\langle\mathbf{B}(\mathbf{r}) \mathbf{B}(0)\rangle
$$

If magnetic fields diffuse up to a scale $R$, then the average magnetic field squared which remains after diffusion will be given in terms of the correlation function by

$$
\left\langle B^{2}\right\rangle_{R} \sim\langle C(\mathbf{r})\rangle_{R}
$$

where $\langle\cdots\rangle_{R}$ denotes the spatial average over a volume of size $\sim R^{3}$. From this result we estimate the typical energy in the magnetic field at any time at distance scales greater than the magnetic field diffusion length at that time. Finally we discuss the possible relevance of this work for cosmology. Throughout we use units in which $c=\hbar=1$.

\section{ELECTROWEAK PHASE TRANSITION BUBBLES AND TURBULENCE}

The electroweak phase transition appears, within acceptable parameters, to be weakly

first order [8]- [12]. As the universe cools through the electroweak transition temperature, $T_{\mathrm{c}} \sim 100 \mathrm{GeV}$, the plasma in the unbroken phase supercools; eventually, one assumes, small regions of the broken symmetry phase, with non-zero Higgs expectation value, nucleate, forming bubbles which expand and fill the system [15]- [17].

The typical size of a bubble after the phase transition is completed is in the range

$$
R_{\text {bubble }} \sim f_{\mathrm{b}} H_{\text {ew }}^{-1}
$$


where

$$
H_{\mathrm{ew}}^{-1} \sim \frac{m_{\mathrm{Pl}}}{g_{*}^{1 / 2} T_{\mathrm{c}}^{2}} \sim 10 \mathrm{~cm}
$$

is the size of the event horizon at the electroweak scale, $m_{\mathrm{Pl}}$ is the Planck mass, $g_{*} \sim 10^{2}$ is the number of massless degrees of freedom in the matter, and the fractional size $f_{\mathrm{b}}$ is $\sim 10^{-2}-10^{-3} 15,16$. These numbers are typical of parameters characteristic of the electroweak phase transition.

The bubble wall surface appears to be stable against small fluctuations [17]. The bubble wall velocity, while poorly known, should be in the range

$$
v_{\text {wall }} \sim 0.05-0.9
$$

When $v_{\text {wall }}<1 / \sqrt{3}$, the sound velocity in the symmetric phase of the electroweak plasma, the burning of the symmetric phase proceeds by deflagration in which the phase transition burning front expands out into the symmetric phase. Ahead of the burning front, a supersonic shock moves into the symmetric phase, accelerating it outward, as shown schematically in Fig. 1a. On the other hand, when the bubble wall velocity is supersonic in the symmetric phase, the burning discontinuity generates in its wake a similarity rarefaction wave, with velocity and temperature profiles illustrated in Fig. 1b.

As two shock fronts associated with the nucleation bubbles collide, they generate turbulence, shown in Fig. 2. At the point where the shock fronts intersect, the velocity of the fluid from one bubble is in a different direction than that from the other bubble. The geometry is similar to the classic problem of the formation of a turbulent jet. The Reynolds number, $R e$, of the flow around two colliding bubbles is sufficiently large that we expect fully developed turbulence in a cone associated with the intersection of the two bubbles, whose opening angle depends on the angle of intersection of the two fluids [18.

The Reynolds number for the collision of two bubbles is

$$
R e \sim \frac{v_{\text {fluid }} R_{\text {bubble }}}{\lambda}
$$


where $v_{\text {fluid }}$ is the typical fluid velocity, which we take to be $\sim v_{\text {wall }} \sim 10^{-1}$; the typical size of a bubble, $R_{\text {bubble }}$, is given by Eq. (3). The typical scattering length $\lambda$ of excitations in the plasma is of order

$$
\lambda \sim \frac{1}{T g_{\mathrm{ew}} \alpha_{\mathrm{w}}^{2}\left|\ln \alpha_{\mathrm{w}}\right|},
$$

where $\alpha_{\mathrm{w}}$ is the fine structure constant at the electroweak scale, and $g_{\text {ew }} \sim g_{*}$ is the number of degrees of freedom that scatter by electroweak processes [19]. Therefore

$$
R e \sim 10^{-3} \frac{m_{\mathrm{Pl}}}{T_{\mathrm{c}}} \alpha_{\mathrm{w}}^{2}\left|\ln \alpha_{\mathrm{w}}\right| \sim 10^{12}
$$

is sufficiently huge, for any macroscopic size bubble, that the collision of the shocked matter should generate turbulent flow.

A turbulent conducting fluid develops magnetic turbulence, resulting in magnetic fields on all scale sizes. The relevant time scale for the amplification of fields on length scale $l$ is of order $\left(l / R_{\text {bubble }}\right) t_{\text {trans }}$, where $t_{\text {trans }} \sim R_{\text {bubble }} / v_{\text {wall }}$ is the duration of the phase transition. If the field growth is exponential, fields on scales $l \lesssim R_{\text {bubble }}$ can be amplified by many $e$-folds. When magnetic turbulence becomes fully developed, the kinetic energy of the turbulent flow is equipartitioned with that of the magnetic field energy, implying that the magnetic fields, $B\left(R_{\text {bubble }}\right)$, generated on the scale of the phase transition bubbles, the largest scale typical of the turbulent flow, are given by

$$
B^{2}\left(R_{\text {bubble }}\right) \sim \epsilon\left(T_{\mathrm{c}}\right) v_{\text {fluid }}^{2}
$$

where $\epsilon\left(T_{\mathrm{c}}\right) \sim g_{*} T_{\mathrm{c}}^{4}$ is the energy density of the electroweak plasma. Since the velocity of the turbulent flow is $\sim 10^{-1}$, a very large fraction of the energy of the fluid is in electromagnetic fields. Turbulent flow is therefore capable of amplifying any seed field by many orders of magnitude.

Thermal fluctuations provide a omnipresent source of seed fields whose size can be estimated by Eqs. (1) and (2), with the average in Eq. (1) the thermal average for a free photon gas. For scale $R \gg T_{\mathrm{c}}^{-1}$ one has 


$$
B_{\text {fluct }}^{2} \sim \frac{T_{\mathrm{c}}}{R^{3}} \sim g_{*}^{3 / 2} T_{\mathrm{c}}^{4} f_{\mathrm{b}}^{-3}\left(\frac{T_{\mathrm{c}}}{m_{\mathrm{Pl}}}\right)^{3}\left(\frac{R_{\text {bubble }}}{R}\right)^{3},
$$

i.e., for $R \sim R_{\text {bubble }}$ the value of $B_{\text {fluct }}^{2}$ is smaller than the equipartition value by a factor of $\left(g_{*}^{1 / 2} / v_{\text {fluid }}^{2}\right) f_{\mathrm{b}}^{-3}\left(T_{\mathrm{c}} / m_{\mathrm{Pl}}\right)^{3}$.

\section{MAGNETIC FIELD GENERATED BY DIPOLE CHARGE LAYER IN THE BUBBLE WALL}

Let us now compare the magnitude of magnetic fields generated by turbulence with those generated from currents in the bubble walls in the absence of magnetic turbulence. These currents arise from the electric dipole layer that develops in the bubble wall, as a consequence of the baryon asymmetry of matter undergoing the transition combined with the fact that the mass of the top quark is comparable to the phase transition temperature.

The net baryon number is non-zero in the broken symmetry phase. If baryogenesis is not associated with the electroweak phase transition, then the local baryon density in the neighborhood of the bubble wall is characterized by a baryon number chemical potential $\mu_{b} \sim 10^{-9} T_{\mathrm{c}}$. On the other hand, if the baryon asymmetry is generated at the electroweak phase transition, the asymmetry is driven by $\mathrm{CP}$ violating effects and $\mu_{b}$ can be much larger. We take as an acceptable range $10^{-9}<\mu_{b} / T_{\mathrm{c}}<10^{-2}$.

The expectation value of the Higgs field goes from zero to a finite value as one traverses the bubble wall into the broken symmetry phase. Thus the top quark has a non-zero mass inside the bubble, and zero mass outside. Top quarks present outside the bubbles face a potential barrier at the bubble wall, which leads to a Boltzmann suppression of their number inside the bubble and near the surface. The slight excess of top over anti-top quarks contributes a net positive charge outside the bubbles, but the charge excess from the tops is suppressed inside. Overall charge neutrality is guaranteed by the presence of a small electrostatic potential, $A^{0}$, which is more negative inside the bubble than outside, attracting light positively charged particles to the interior. 
The electrostatic potential is determined by

$$
-\nabla^{2} A^{0}=e \rho_{\mathrm{em}}(x)
$$

where $\rho_{\mathrm{em}}$ is the total local electric charge. Expanding the distributions of the various charged species to leading order in $A^{0}$ and the small non-zero charge, baryon and lepton number chemical potentials, and then for simplicity expanding in powers of $\left(m_{\mathrm{top}} / T\right)^{2}$, we find

$$
e \rho_{\mathrm{em}}+m_{\mathrm{D}}^{2} A^{0} \sim-e m_{\mathrm{top}}^{2}(x) \mu_{b}
$$

where $m_{\mathrm{D}}$ is the Debye mass for massless leptons and quarks (including the top). (We neglect a small correction to the Debye screening term $\sim m_{\text {top }}^{2}(x)$.) The Debye screening length $1 / m_{\mathrm{D}}$ is of order $1 / e T$. In the expected limit where the thickness, $L_{\text {wall }}$, of the bubble wall is $\gg 1 / m_{\mathrm{D}}$, we solve Eqs. (11) and (12) by expanding in powers of $\nabla^{2} / m_{\mathrm{D}}^{2}$, and find

$$
e \rho_{\mathrm{em}}(x) \sim \frac{e \mu_{b}}{m_{\mathrm{D}}^{2}} \nabla^{2} m_{\mathrm{top}}^{2}(x)
$$

plus corrections involving higher order powers of $1 /\left(m_{\mathrm{D}} L_{\text {wall }}\right)^{2}$. The charge density on the surface, $x \approx 0$, is of order

$$
e \rho_{\mathrm{em}}(0) \sim \frac{1}{e} \eta_{b} T_{\mathrm{c}}^{3} \varepsilon
$$

where the typical baryon asymmetry near the bubble wall is $\eta_{b} \sim \mu_{b} / T_{\mathrm{c}}, m_{D}^{2} \sim e^{2} T_{\mathrm{c}}^{2}$, and

$$
\varepsilon \equiv\left(\frac{m_{\mathrm{top}}}{T_{\mathrm{c}}^{2} L_{\mathrm{wall}}}\right)^{2} .
$$

Typically $T_{\mathrm{c}} L_{\text {wall }} \sim 10-100$, so that $\varepsilon \sim 10^{-2}-10^{-4}$. For $m_{\text {top }}(x)$ monotonically decreasing from inside to out across the bubble surface, we see that the bubble has a dipole charge layer of order $e \rho_{\mathrm{em}}(0) L_{\mathrm{wall}}$ per unit area.

In order for the dipole charge layer on the bubble wall to generate a magnetic field the bubble must have a net rotation. If the bubble is propagating in a turbulent region with vorticity, its surface acquires a net rotational velocity, $v_{\text {rot }}$, of order the typical turbulent 
velocity in the fluid, $v_{\text {fluid }} \sim 10^{-1}$. A rotating bubble thus has associated with it a magnetic moment of order

$$
\begin{aligned}
M_{\text {bubble }} & \sim e \rho_{\mathrm{em}} L_{\text {wall }}^{2} R_{\text {bubble }}^{2} v_{\text {rot }} \\
& \sim \eta_{b} \frac{m_{\text {top }}^{2}}{m_{\mathrm{D}}} v_{\text {fluid }} R_{\text {bubble }}^{2}
\end{aligned}
$$

The magnetic field generated by the bubble on a scale size of order the bubble radius is therefore

$$
\begin{aligned}
B_{\text {bubble }} & \sim \frac{M_{\text {bubble }}}{R_{\text {bubble }}^{3}} \sim \eta_{b} \frac{m_{\text {top }}^{2} v_{\text {fluid }}}{m_{\mathrm{D}} R_{\text {bubble }}} \\
& \sim g_{*}^{1 / 2} v_{\text {fluid }} T_{\mathrm{c}}^{2} \frac{\eta_{b}}{f_{\mathrm{b}}} \frac{m_{\text {top }}^{2}}{m_{\mathrm{D}} m_{\mathrm{Pl}}} .
\end{aligned}
$$

We note that the magnetic field produced by this mechanism is smaller by a factor $\sim$ $\eta_{b} T_{\mathrm{c}} / m_{\mathrm{Pl}}$ compared with the field expected from equipartition in a magnetically turbulent environment, Eq. (9). At the electroweak scale the equipartition field is $\sim 10^{24}$ gauss, and the field produced from the dipole layers in the bubble walls, $\sim 10^{-2}$ gauss, is a factor $\sim 10^{-26}$ smaller.

Cheng and Olinto [7], by contrast, find a field produced by the dipole layer in the bubbles at the QCD transition, at $T \sim 100 \mathrm{MeV}$, of order $10^{-10}-10^{-12}$ of the equipartition field, $\sim 10^{18}$ gauss. The reason for their much larger fraction is that they assume that the width

of the dipole layer is controlled by the width of the baryon diffusion layer, $\sim 10^{7} \mathrm{fm}$, rather than the microphysics length scale $\sim 1 \mathrm{fm}$ at the QCD scale. The diffusion layer for the baryon number is so much larger because the QCD transition occurs sufficiently slowly that the particles have time to undergo many scatterings during the transition. In the electroweak transition on the other hand, the bubble walls move relativistically, and little diffusion takes place.

\section{LARGE SCALE MAGNETIC FIELDS}

We now ask how magnetic fields on scales larger than the electroweak bubble size are generated by superposition of the fields associated with the turbulent flow. A first estimate 
can be made by assuming that the magnetic fields on scales large compared with the size of the turbulent velocity fields are generated by randomly oriented magnetic dipoles of typical size that of this velocity field.

A magnetic dipole $M$ generates a magnetic field far from the dipole of order

$$
B(\vec{r}) \sim e \frac{M}{\left|\mathbf{r}-\mathbf{r}_{\mathrm{d}}\right|^{3}}
$$

where $\mathbf{r}_{\mathrm{d}}$ is the position of the dipole. Near the dipole the field saturates to a more or less constant value. To estimate the net magnetic fields produced by randomly oriented dipoles, we make a continuum approximation for the distribution of dipoles, and assume that the density $\nu^{i}(\vec{r})$ of dipoles pointing in the $i$ th direction is Gaussianly distributed with measure

$$
\int\left[d \nu^{i}\right] \exp \left\{-\frac{1}{2 \kappa} \int d^{3} r \vec{\nu}^{2}(\mathbf{r})\right\},
$$

where $\kappa$ is a constant. The correlation function of the density of dipoles implied by the distribution (19) is

$$
\left\langle\nu^{i}(\mathbf{r}) \nu^{j}(0)\right\rangle=\kappa \delta^{i j} \delta^{(3)}(\mathbf{r})
$$

From Eqs. (18) and (20) we thus find the magnetic field-magnetic field correlation function

$$
\langle\mathbf{B}(\mathbf{r}) \cdot \mathbf{B}(\mathbf{0})\rangle \sim e^{2} \kappa \int d^{3} r_{\mathrm{d}} \frac{1}{\left|\mathbf{r}-\mathbf{r}_{\mathrm{d}}\right|^{3}} \frac{1}{\left|\mathbf{r}_{\mathrm{d}}\right|^{3}} .
$$

This integral representation is dominated by the regions where either $\left|\mathbf{r}_{\mathrm{d}}\right| \rightarrow 0$ or $\left|\mathbf{r}-\mathbf{r}_{\mathrm{d}}\right| \rightarrow$ 0 . The logarithmic divergence of the integral in these regions is cut off by the size of the typical dipole, $f_{\mathrm{b}} H_{\mathrm{ew}}^{-1}$, so that for $r \gg f_{\mathrm{b}} H_{\mathrm{ew}}^{-1}$,

$$
\langle\mathbf{B}(\mathbf{r}) \cdot \mathbf{B}(\mathbf{0})\rangle \sim \frac{e^{2} \kappa}{r^{3}} \ln \left(\frac{H_{\mathrm{ew}} r}{f_{\mathrm{b}}}\right) .
$$

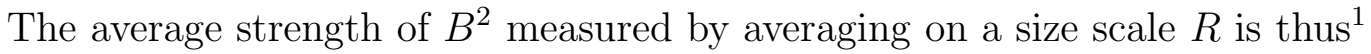

\footnotetext{
${ }^{1}$ This results differs from Hogan [4], who on the basis of a random walk of the field lines finds $\left\langle B^{2}\right\rangle_{R} \sim R^{-3}$.
} 


$$
\left\langle B^{2}\right\rangle_{R} \sim \frac{e^{2} \kappa}{R^{3}} \ln ^{2}\left(\frac{H_{\mathrm{ew}} R}{f_{\mathrm{b}}}\right) .
$$

If we assume equipartition, and compare with Eq. (9), we see that $\kappa$ is given by

$$
e^{2} \kappa \sim\left(f_{\mathrm{b}} H_{\mathrm{ew}}^{-1}\right)^{3} \epsilon v_{\text {fluid }}^{2}
$$

and therefore

$$
\left\langle B^{2}\right\rangle_{R} \sim v_{\text {fluid }}^{2} g_{*} T_{\mathrm{c}}^{4}\left(\frac{f_{\mathrm{b}}}{H_{\mathrm{ew}} R}\right)^{3} \ln ^{2}\left(\frac{H \mathrm{ew} R}{f_{\mathrm{b}}}\right) .
$$

The expansion of the universe causes the magnetic field to decrease as the square of scale factor of the universe. Therefore, in the absence of flux diffusion, the ratio of $\left\langle B^{2}\right\rangle$ to the energy $\rho_{\gamma}$ in photons,

$$
\eta_{B} \equiv \frac{\left\langle B^{2}\right\rangle_{R}}{\rho_{\gamma}}
$$

remains independent of time, when measured on a comoving scale size. From (25) we have

$$
\eta_{B} \sim v_{\text {fluid }}^{2} f_{\mathrm{b}}^{3}\left(\frac{\lambda_{\mathrm{ew}}}{R}\right)^{3} \ln ^{2}\left(\frac{R}{f_{\mathrm{b}} \lambda_{\text {ew }}}\right),
$$

where $\lambda_{\text {ew }}$ is the size of the universe at the electroweak phase transition times the scale factor, $T_{\mathrm{c}} / T_{\gamma}$, where $T_{\gamma}$ is the temperature of the microwave background; at the present epoch $\lambda_{\text {ew }} \sim 10^{2} \mathrm{AU}$.

Magnetic fields from the electroweak transition can survive only on scales on which magnetic diffusion has not had time to wash out the field correlations. The flux diffusion equation, which in the local fluid rest frame can be written as,

$$
\partial_{t}^{2} \mathbf{B}+k^{2} \mathbf{B}+4 \pi \sigma \partial_{t} \mathbf{B}=0
$$

where $\sigma$ is the electrical conductivity, implies that a magnetic field of length scale $1 / k$ dies off on a time scale

$$
\tau \sim \sigma / k^{2}
$$


long wavelength magnetic fields set up at the electroweak phase transition die away very slowly.

The characteristic diffusion distance at time $\tau$ is therefore of order

$$
l_{\mathrm{diff}} \sim \sqrt{\tau / \sigma}
$$

at $\tau$ magnetic fields on length scales larger than $l_{\text {diff }}$ are not yet significantly affected by diffusion. An upper limit on the diffusion length is its present value, about 3 AU [7] which is not much smaller than the size of the horizon at the electroweak phase transition.

In the present epoch, $\eta_{B} \sim\left(10^{-6}-10^{-9}\right) \times\left(10^{2} \mathrm{AU} / R\right)^{3} \ln ^{2}(R / 1 \mathrm{AU})$ for $f_{b}=10^{-2}-$

$10^{-3}$. Thus the equipartition magnetic field on a scale of order of diffusion length in the extragalactic medium is now about $B(R \sim 10 \mathrm{AU}) \sim 10^{-7}-10^{-9}$ gauss. These fields might have acted in an earlier epoch as seed fields, subsequently ampified by a galactic dynamo mechanism to produce the galactic magnetic fields. On a galactic size scale, at present seven orders of magnitude larger than the present size of the electroweak horizon, one finds that the equipartition field is $B\left(R \sim 10^{9} \mathrm{AU}\right) \sim 10^{-17}-10^{-20}$ gauss.

\section{ACKNOWLEDGMENTS}

We are grateful to Baolian Cheng for stimulating our interest in this problem, and to Angela Olinto for subsequent discussions. This research was supported by the U.S. Department of Energy under Grants No. DOE High Energy DE-AC02-83ER40105 and No. DOE Nuclear DE-FG02-87ER-40328, and NSF Grants PHY 89-21025 and PHY 94-21309. GB and LM are grateful for the hospitality of the Los Alamos National Laboratory during the time that this work was initiated, and of the Aspen Center for Physics where this work was completed. The work of DB has been supported by the Deutsche Forschungsgemeinschaft. 


\section{REFERENCES}

[1] E. N. Parker, Cosmical Magnetic Fields (Oxford Univ. Press, Oxford, 1979).

[2] Ya. B. Zel'dovich, A. A. Ruzmaikin, and D. D. Sokoloff, Magnetic Fields in Astrophysics (Gordon and Breach, New York, 1983); The Almighty Chance (World Scientific, Singapore, 1990).

[3] M. S. Turner and L. Widrow, Phys. Rev. D37, 2743 (1988).

[4] C. J. Hogan, Phys. Rev. Lett. 51, 1488 (1983).

[5] T. Vachaspati, Phys. Lett. B265, 258 (1991).

[6] J. M. Quashnock, A. Loeb, and D. N. Spergel, Astrophys. J. 344, L49 (1989).

[7] B. Cheng and A. Olinto, Phys. Rev. D50, 2421 (1994).

[8] D.A. Kirzhnits and A.D. Linde, Ann.Phys. 101, 195 (1976); A.D. Linde, Phys. Rev. D14, 3345 (1976).

[9] M. E. Carrington, Phys. Rev. D45, 2933 (1992).

[10] G. W. Anderson and L. J. Hall, Phys. Rev. D45, 2685 (1992).

[11] M. Dine, R. G. Leigh, P. Huet, A. Linde, and D. Linde, Phys. Rev. D46, 550 (1992).

[12] K. Farakos, K. Kajantie, K. Rummukainen, and M. Shaposhnikov, CERN-TH-7220-94, hep-lat/9412091.

[13] N. Goldenfeld, in Proc. NATO ARW on "Formation and Interactions of Topological Defects," A. C. Davis and R. H. Brandenberger (eds). (Plenum Press, N. Y., 1995). (University of Illinois preprint $\mathrm{P}=95-01-002$.)

[14] E. M. Lifshitz and L. P. Pitaevskii, Physical Kinetics, (Pergamon, New York, 1981).

[15] N. Turok, Phys. Rev. Lett. 68, 1803 (1992). 
[16] B. Liu, L. McLerran, and N. Turok, Phys. Rev. D46, 2668 (1992).

[17] P. Huet, K. Kajantie, R. G. Leigh, B. Liu, and L. McLerran, Phys. Rev. D48, 2477 (1993).

[18] L. D. Landau and E. M. Lifshitz, Fluid Mechanics (Pergamon Press, New York, 1959).

[19] G. Baym, H. Monien, C.J. Pethick, and D.G. Ravenhall, Phys. Rev. Lett. 64, 1867 (1990); H. Heiselberg, G. Baym, C.J. Pethick, and J. Popp, Nucl. Phys. A544, 569c (1992). 


\section{FIGURES}

FIG. 1. Bubble structure in $(1+1)$ dimensions for a first-order electroweak phase transition. The energy density $\epsilon$ and the fluid rapidity $\Theta=\ln \left[\left(1+v_{\text {fluid }}\right) /\left(1-v_{\text {fluid }}\right)\right]$ are plotted versus the space-time rapidity $y=\ln [(t+x) /(t-x)]$. (a) Deflagration bubble; $\Theta_{\text {def }}$ and $\Theta_{\text {sh }}$ denote the rapidities of the deflagration front and the shock front, respectively. (b) Detonation bubble; here $\Theta_{\mathrm{s}}$ is the rapidity corresponding to the sound velocity and $\Theta_{\text {det }}$ denotes the rapidity of the detonation front.

FIG. 2. Fluid velocities during the collision of two bubbles. The dashed line represents the shock (detonation) front for a deflagration (detonation) bubble. 


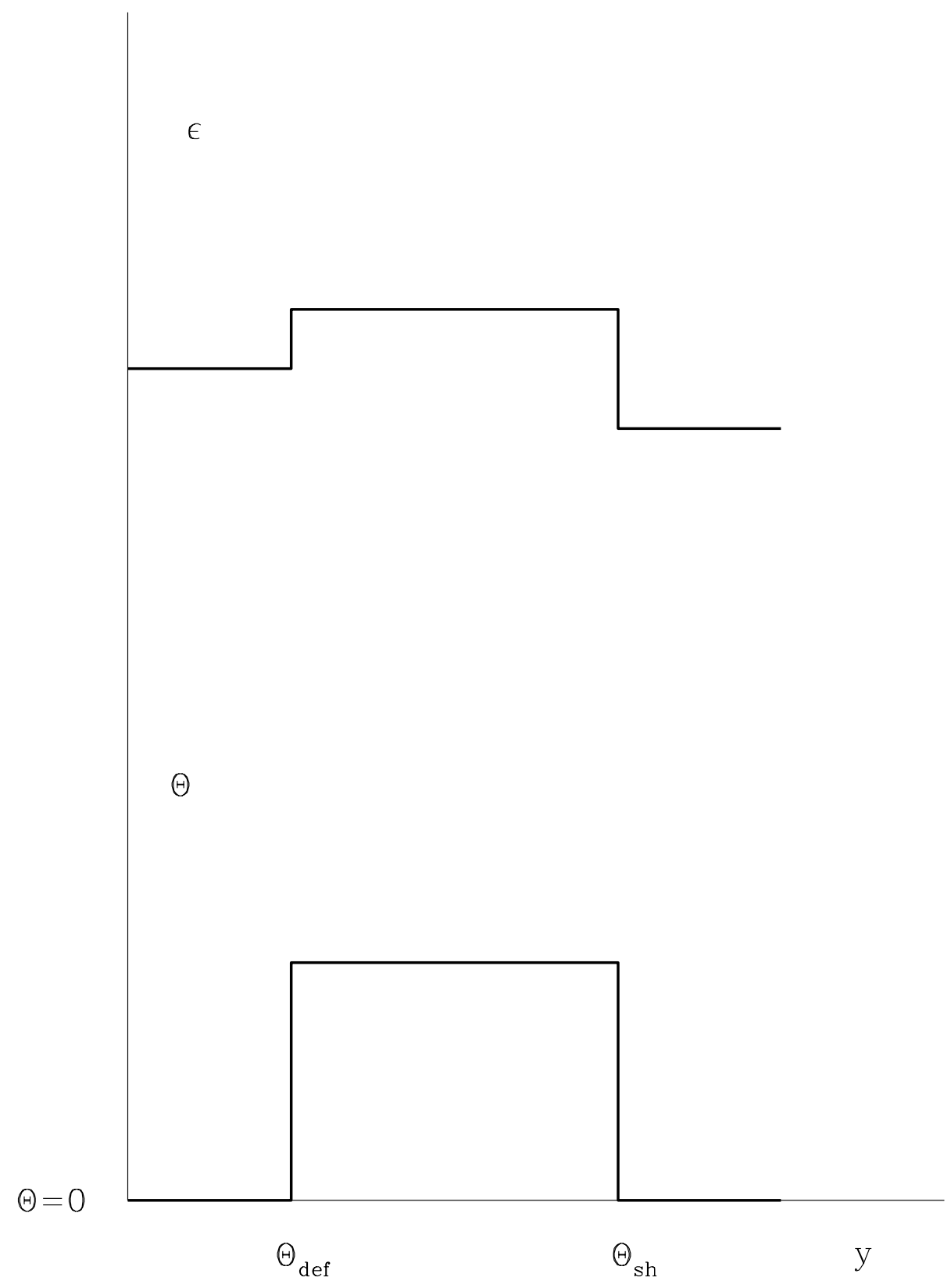

Figure 1 a 


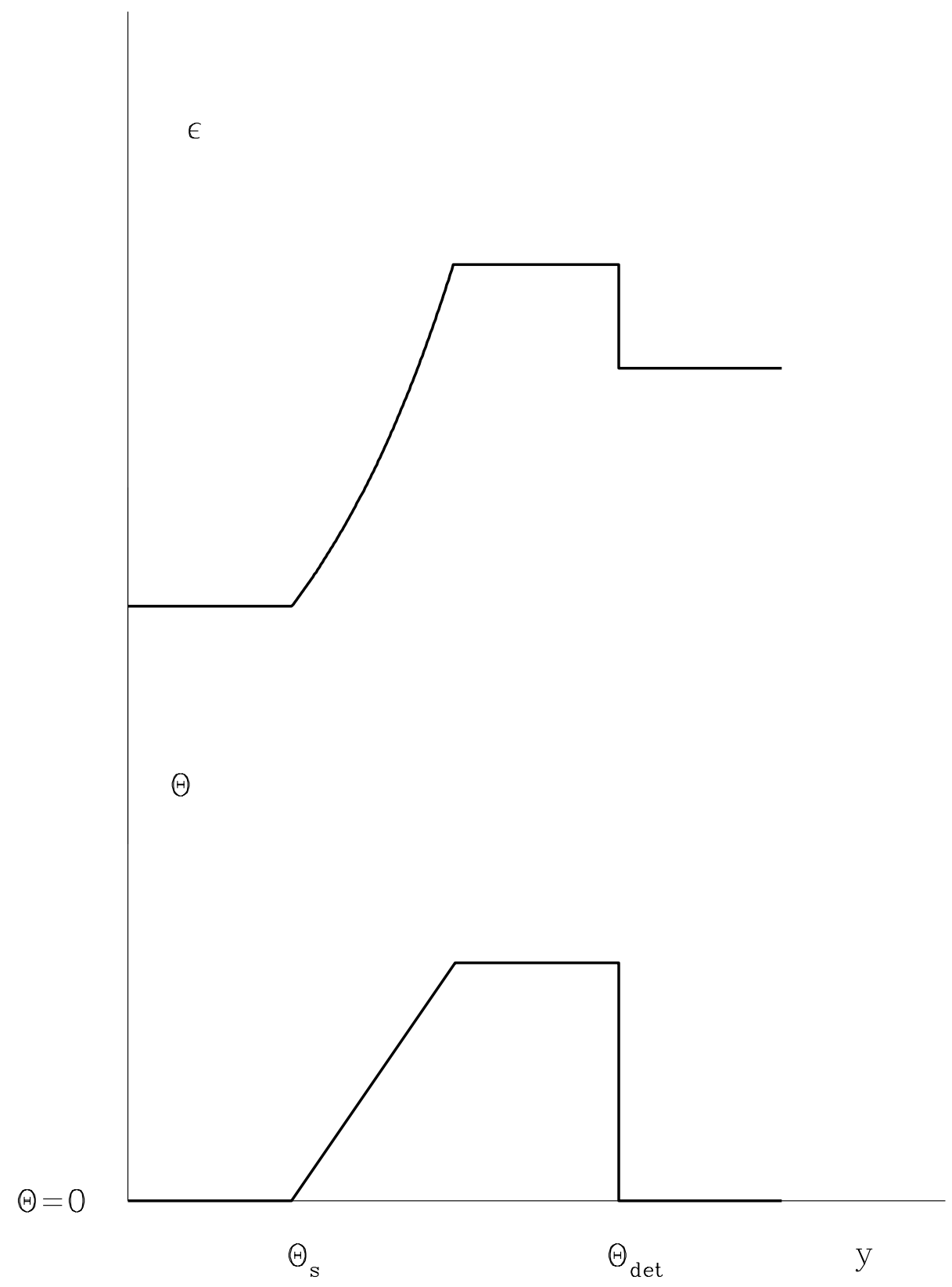

Figure 1b 


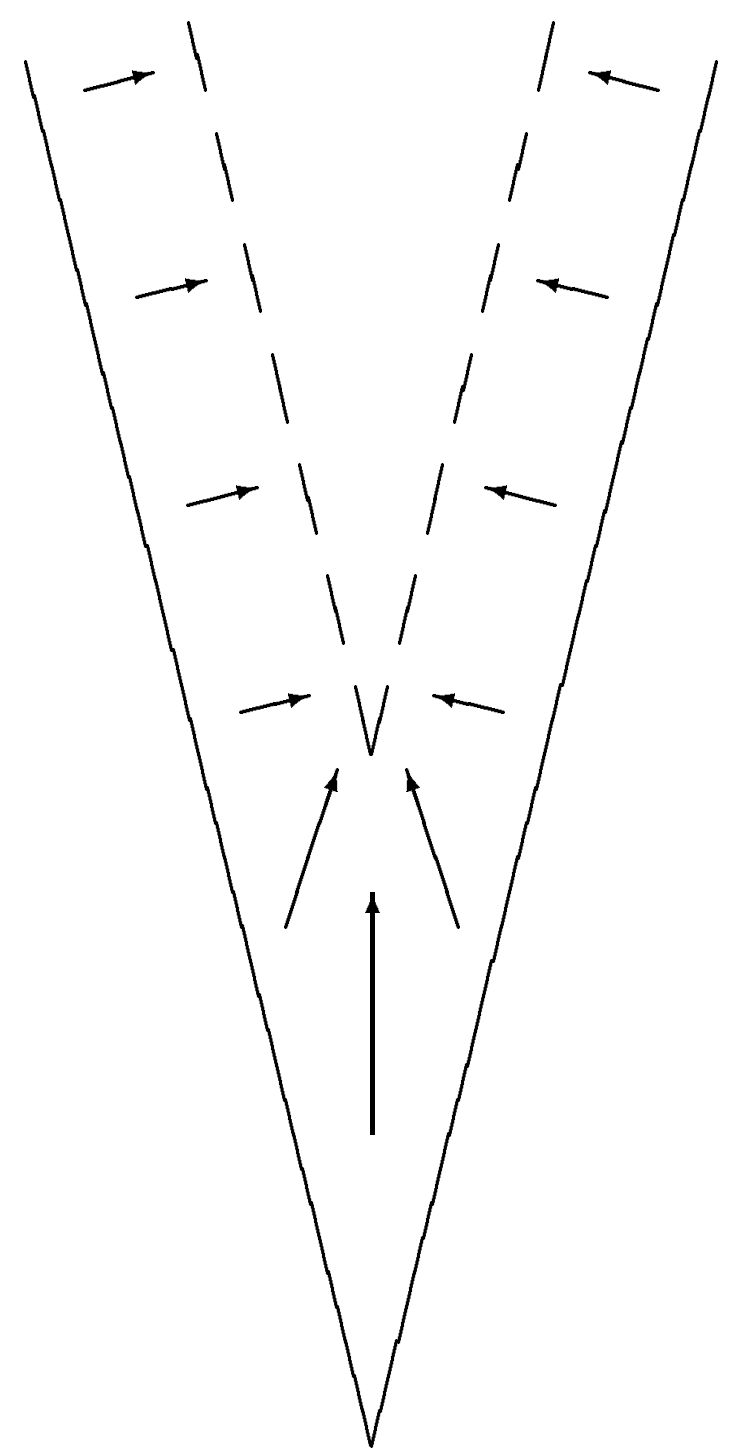

Figure 2 